\title{
Intranasale Therapie wirkt in den Augen
}

\author{
Glukokortikoide werden seit langem erfolgreich bei allergischen \\ Erkrankungen eingesetzt. Eine neue Fluticasonverbindung erweist \\ sich in der Behandlung der allergischen Rhinokonjunktivitis als \\ wirksam und verträglich.
}

ntranasale Glukokortikoide werden

besonders bei moderaten bis schweren Formen der allergischen Rhinitis eingesetzt, vor allem wenn die nasale Kongestion im Vordergrund steht. Entsprechende Empfehlungen geben die Therapieleitlinien der American Academy of Allergy, Asthma and Immunology beziehungsweise die Leitlinien der Initiative Allergic Rhinitis and its Impact on Asthma (ARIA). Mit Fluticasonfuroat steht ein neues lokal wirksames Glukokortikoid für die Rhinitistherapie zur Verfügung.

In einer Dosisfindungsstudie erwies sich die einmalige Applikation von 110 $\mu \mathrm{g}$ als optimal. In einer randomisierten doppelblinden und plazebokontrollierten Phase-III-Studie wurden Wirksamkeit und Verträglichkeit von Fluticason- furoat bei 299 Erwachsenen und Jugendlichen ab zwölf Jahren mit allergischer Ragweed-Rhinitis überprüft. Dabei ergab sich während der zweiwöchigen Behandlung mit dem Verum-Spray eine signifikant größere Verbesserung des na-

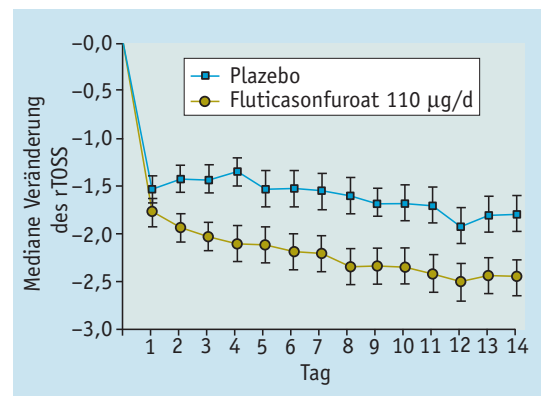

Okuläre Symptome verringern sich bei intranasaler Therapie. salen Gesamtsymptomscores tagsüber, des morgendlichen nasalen Gesamtsymptomscores vor der Sprayapplikation und des täglichen okulären Gesamtsymptomscores im Vergleich zu Plazebo. Der symptomlindernde Effekt trat acht Stunden nach der Erstapplikation des Sprays auf. Die Behandlung wurde gut vertragen, lediglich Kopfschmerzen wurden in der Verumgruppe etwas häufiger als in der Plazebogruppe berichtet. Eine Besserung allergischer Augensymptome durch Fluticasonfuroat war für die älteren intranasalen Glukokortikoide in klinischen Studien bisher nicht konsistent nachweisbar.

Fazit: Die Behandlung der saisonalen allergischen Rhinitis mit intranasalem Fluticasonfuroat einmal täglich verbesserte signifikant die nasalen und okulären Symptome. Der Effekt trat acht Stunden nach Erstapplikation ein und hielt 24 Stunden an.

Kaiser HB et al. Fluticasone furoate nasal spray: A single treatment option for the symptoms of seasonal allergic rhinitis. J Allergy Clin Immunol 2007; 119: 1430-7

\section{Lebensqualität bei Rhinitis erfassen}

\section{Atemwegsallergien beeinträchtigen nicht nur die physische, sondern auch die psychische Funktionsfähigkeit. Generische Fragebögen zur Lebensqualität liefern dazu eventuell detailliertere Informationen als krankheitsbezogene Lebensqualitätsfragebögen.}

ie Erfassung der Lebensqualität (LQ) bei Patienten mit einer Rhinokonjunktivitis (RC) erfolgt meist mit dem krankheitsbezogenen RC-Lebensqualitätsfragebogen nach Juniper (RQLQ). Generische LQ-Fragebögen erfassen auch Aspekte über die spezifischen Krankheitsprobleme hinaus. Der 15D-Lebensqualitätsfragebogen erfasst 15 Dimensionen wie z.B. Mobilität, Schlaf, Essverhalten, Aktivitäten, mentale Funktion oder sexuelle Aktivitäten.

RQLQ und 15D-Fragebogen wurden jetzt in einer Observationsstudie mit gräser- und milbenallergischen Patienten direkt miteinander verglichen. Dazu ga- ben 248 Patienten mit einer RC und 121 Patienten mit einer RC und Asthma unmittelbar vor Beginn einer SIT aus ihrer Erinnerung heraus Auskunft über ihre allergischen Symptome während eines typischen Krankheitstages (RQLQ) bzw. ihre Gesundheit an Tagen mit oder ohne Symptome (15D).

RC-Patienten mit persistierender mäßiger bis schwerer Erkrankung berichteten über eine verminderte Lebensqualität in allen Punkten des RQLQ während der Allergenexposition. Auch der durchschnittliche Score im 15DFragebogen war an Tagen mit Allergie verringert ( 0,83 vs. 0,98 an Tagen ohne
Allergie, $\mathrm{p}<0,001)$. An Tagen mit allergischen Symptomen bestand eine signifikante Korrelation zwischen den Ergebnissen des 15D-Fragebogens und dem RQLQ ( $r=-0,42, p<0,001)$. Signifikante Differenzen in der gesundheitsbezogenen Lebensqualität zwischen Patienten mit oder ohne Asthma waren nur mit dem 15D-Fragebogen feststellbar.

Fazit: Bei Patienten mit allergischer RC lässt sich sowohl mit Hilfe des krankheitsspezifischen RQLQ als auch des generischen 15D-Fragebogens eine Verschlechterung der Lebensqualität bei Allergenexposition feststellen. Der 15DFragebogen scheint dabei aber einen umfassenderen Einblick in die Veränderung der Lebensqualität bei Allergenexposition zu geben als der RQLQ. $\quad b k$

Petersen KD et al. Quality of life in rhinoconjunctivitis assessed with generic and disease-specific questionnaires. Allergy 2008; 63: 284-91 